\title{
Cocoon Characteristics of Antheraea pernyi Silkworm Reared in Korean Oak Field
}

\author{
Bong-Seob Shin, Jong-Young Jeon, and Jong-Ho Kim* \\ School of Textile Engineering and Fashion Design, Kyungpook National University, Sangju, 742-711, Korea
}

(Received 6 November 2012; Accepted 27 December 2012)

\begin{abstract}
Antheraea pernyi silkworm is a well known wild silkworm to produce a valuable silk fiber. $A$. pernyi silkworm was reared in Korean oak field and examined the cocoon characteristics, such as cocoon weight, cocoon shell weight, and percentage of cocoon shell weight. Degumming loss was also measured after alkali degumming process. $A$. pernyi silkworm spins tawny color cocoon in oval shape. Cocoon shell weight of $A$. pernyi silkworm, $0.78 \mathrm{~g}$, was heavier than that of $B$. mori silkworm, 0.51 to $0.63 \mathrm{~g}$. Cocoon shell percentage of $A$. pernyi silkworm, $32.8 \%$, was higher than that of $B$. mori silkworm, 23.4 to $25.2 \%$. Degumming loss percentage of $A$. pernyi silkworm, $17.1 \%$, was lower than that of $B$. mori silkworm, 25.0\%. SEM showed that the surface of the cocoon filament was coarse and oriented with longitudinal direction.
\end{abstract}

Key words: Antheraea pernyi, Cocoon trait, Cocoon shape, Degumming loss

\section{Introduction}

Cocoon has been used as a source of textile fiber in the world. It is spun by various kinds of silkworm; domestic silkworm, Bombyx mori, and wild silkworm, Antheraea species. Among them, Antheraea pernyi silkworm is one of the most useful insect to produce valuable textile fiber. In general, $A$. pernyi silkworm spun tawny color cocoon. The tawny color of pernyi cocoon is due to the crosslinked polymer of gentisic acid and silk protein (Tao et al.,

\footnotetext{
*To whom the correspondence addressed

School of Textile Engineering and Fashion Design, Kyungpook National University, Sangju, 742-711, Korea Tel: +82-54-530-5280; E-mail: jong@knu.ac.kr http://dx.doi.org/10.7852/ijie.2012.25.2.205
}

1993). In Korea, $A$. pernyi silkworm has been reared until 1964 (Moon, 2000). Silk protein spun by $A$. pernyi is more stable against chemicals than that spun by $B$. mori silkworm (Kweon et al., 1998). Now a day, A. pernyi silk fibroin had been studied as a non-textile resource in various fields. Dissolution method of $A$. pernyi silk fibroin using various salts and characterized regenerated silk fibroin were reported by Kweon's research group (Kweon et al., 2002; Kweon et al., 2003; Woo et al., 2000). Various types of architecture from pernyi fibroin has been reported such as nanofibers, scaffold, powder, and gel (He et al., 2011; Zhao et al., 2011; Lee et al., 2011; Yan et al., 2010). Lee et al., (2011) reported that hydrolyzed A. per$n y i$ silk fibroin was separated into two parts: alanine-rich fraction and tyrosine-rich fraction. Wild silk fibroin hydrolysate enhances lipid metabolism and antioxidant defense (Um et al., 2011).

Though pernyi silk fibroin has been studied as non-textile materials such as food ingredient and biomaterials, the cocoon characteristics of $A$. pernyi silkworm cocoon has not been reported. Therefore, the authors examined cocoon characteristics of $A$. pernyi silkworm as a basic study for $A$. pernyi silk fibroin.

\section{Materials and Methods}

\section{Materials}

Wild silkworm cocoon, A. pernyi silkworm cocoon was harvested from oak field, National Academy of Agricultural Science, Suwon, Korea. The number of harvested one is 189 in 2009. Sodium carbonate and other chemicals were purchased and used without further purification.

\section{Examination of $\boldsymbol{A}$. pernyi silkworm cocoon}

Each cocoon was weighed to calculate whole cocoon weight, cocoon shell weight, and percentage of cocoon shell weight. Thickness of the cocoon shell was measured 
with vernier calipers. The surface morphology of cocoon filament was observed using Field-Emission Scanning Electron Microscope (SUPRA 55VP, Carl Zeiss, Germany) after platinum coating.

\section{Degumming}

Wild cocoon was sliced and degummed as following method. One hundred fifty grams of cocoon was soaked into the degumming solution (sodium carbonate $2.5 \mathrm{~g} / \mathrm{L}$ ) at boiling temperature for 30 minutes and then washed with distilled water. The degumming process was repeated three times. Degumming loss percentage was calculated according to the following equation.

Degumming loss percentage $(\%)=(\mathrm{W} i-\mathrm{W} f) / \mathrm{W} i * 100$

Where, $\mathrm{W} i$ is initial weight of dry cocoon; $\mathrm{W} f$, final weight of dry cocoon.

\section{Results and Discussion}

\section{A. pernyi silkworm cocoon}

Oak silkworm is distributed on the far-east area including Korea, Japan, and northeastern China. There are two kinds of silkworms, A. pernyi and A. yamamai. Silkworm A. yamamai spins bright green color cocoon and fiber. However, A. pernyi silkworm makes a cocoon yellowish color shown in Fig. 1. The silkworms are fed on plantations of specially trimmed oak trees in National Academy of Agricultural Science. A. pernyi cocoon was harvested in 2009 and stored in conditioned room with low humidity and temperature.

The cocoons are oval in shape. The surface of cocoon is printed by oak leaf. After taking out the pupa, the thickness of the cocoon shell was measured. The thickness of the $A$. pernyi silkworm cocoon shell was $0.409 \pm 0.101$ $\mathrm{mm}$. On the other hand, that of $B$. mori silkworm cocoon was $0.517 \pm 0.077 \mathrm{~mm}$. The thickness of the cocoon shell of $A$. pernyi silkworm was thinner than that of the authorized B. mori silkworm, YangWonJam.
The most important traits economically for silk production are single cocoon weight, cocoon shell weight, and cocoon shell percentage. Table 1 showed the cocoon traits of $A$. pernyi cocoon compared with $B$. mori cocoons. The average weight of $A$. pernyi cocoon completely dried is $2.36 \mathrm{~g}$. To compare the cocoon traits, Bombyx mori silkworm variety, YangWonJam cocoon was harvested from Korean Farmer in 2012 and weighed cocoon characteristics. The reported average dry weights of authorized Bombyx mori cocoon for reeling test were $2.43-2.72 \mathrm{~g}$. The dry weight of our result is similar to those of authorized them. The average weight of $A$. pernyi cocoon shell is $0.78 \mathrm{~g}$. On the other hand, B. mori cocoons showed 0.51

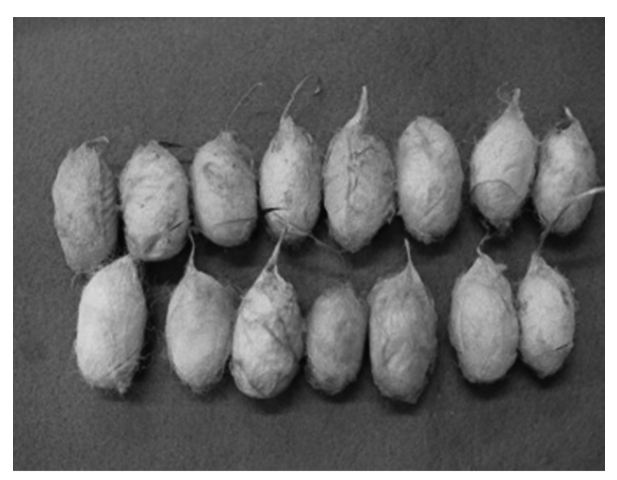

(a)

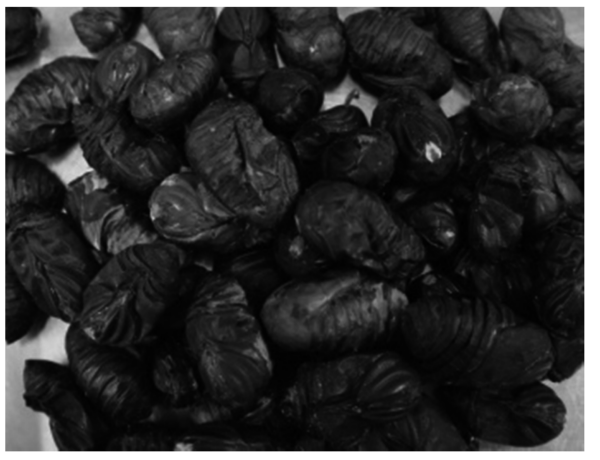

(b)

Fig. 1. Photographs of Antheraea pernyi silkworm cocoon(a), and pupa(b).

Table 1. Cocoon traits of Antheraea pernyi silkworm cocoon

\begin{tabular}{ccccc}
\hline Kinds of cocoon & $\begin{array}{c}\text { Single cocoon weight } \\
(\mathrm{g})\end{array}$ & $\begin{array}{c}\text { Cocoon shell weight } \\
(\mathrm{g})\end{array}$ & $\begin{array}{c}\text { Cocoon shell percentage } \\
(\%)\end{array}$ & Reference \\
\hline Antheraea pernyi & 2.36 & 0.78 & 32.8 & This study \\
YangWonJam & - & 0.51 & 23.4 & This study \\
DaePoongJam & 2.54 & 0.59 & 23.6 & Kang et al., (2012a) \\
DaeBakJam & 2.72 & 0.63 & 23.2 & Kang et al., (2012a) \\
SooOkJam & 2.55 & 0.60 & 23.7 & Kang et al., (2012b) \\
HanSaengJam & 2.43 & 0.61 & 25.2 & Kang et al., (2011) \\
\hline
\end{tabular}


Table 2. Degumming loss percentage of $A$. pernyi silkworm cocoon

\begin{tabular}{cc}
\hline Kinds of cocoon & $\begin{array}{c}\text { Degumming loss } \\
\text { percentage }\end{array}$ \\
\hline Antheraea pernyi silkworm cocoon & 17.1 \\
Bombyx mori silkworm cocoon & 25.0 \\
BaegOkJam fiber* & 25.4 \\
\hline
\end{tabular}

*Kweon et al., (2012)

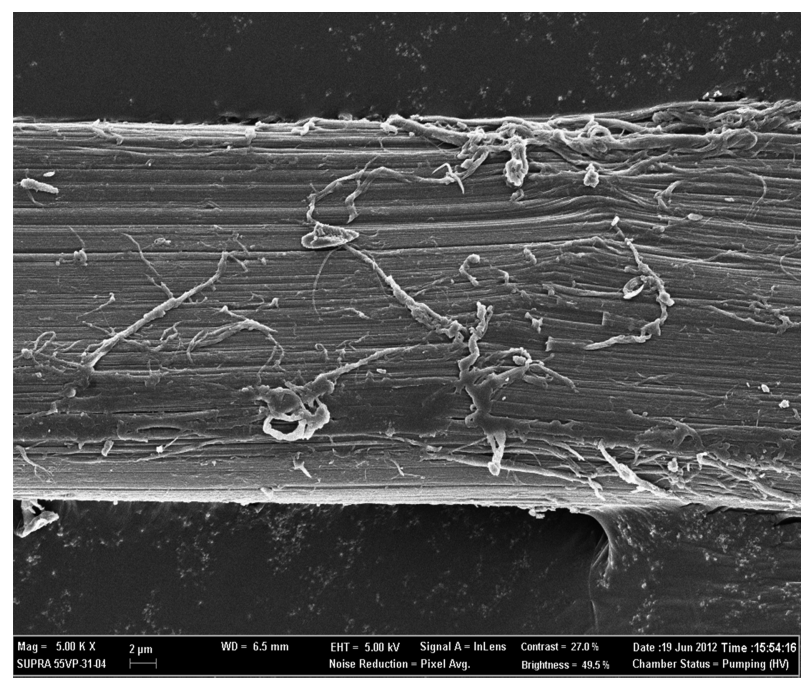

Fig. 2. Scanning electron microscopic photograph of Antheraea pernyi cocoon filament after degumming.

$-0.63 \mathrm{~g}$. These data can be explained by the size of the cocoons because the wild cocoon is larger than domestic ones. Cocoon shell percentage is one of important trait for silk industry. Cocoon shell percentage of $A$. pernyi cocoon is $32.8 \%$. Authorized domestic ones are $23.2-25.2 \%$.

\section{Degumming loss of $A$. pernyi silkworm cocoon}

Silkworm cocoon is composed of fibroin and sericin. Sericin is a gummed material to stick together silk fibroin brins in order to make a single silk filament. Generally, sericin was removed to make good texture of silk fabrics. The weight loss of cocoon filament through degumming process was calculated and showed in Table 1. The degumming loss weights of $A$. pernyi and $B$. mori silkworm cocoon were $17.1 \%$, and $25.0 \%$, respectively.

Kawahara and Nakajima (1992) examined the degumming loss of wild silk fibers with bacterial protease and reported that those of $A$. pernyi, $A$. yamamai, and $A$. assama were $11.6 \%, 16.0 \%$, and $7.4 \%$, respectively. Similarly, those of wild silk fiber with sodium hydrogen carbonate were $11.8 \%$ (A. pernyi), $16.5 \%$ (A. yamamai), and 6.4\% (A. assama). BaegOkJam, an authorized Korean silkworm variety, shows $25.4 \%$ of degumming ratio
(Kweon et al., 2012). Our data were similar to those of the other researchers.

Fig. 2 showed the surface morphology of $A$. pernyi cocoon filament. As shown in Fig. 2, the surface of cocoon filament was coarse and was shown have an orientation with the longitudinal direction. Degummed $A$. pernyi cocoon filament was shown something is attached on the surface of the filament. The attached is might be considered as damaged cocoon filament or sericin remained. According to Kawahara and Nakajima (1992), the surface morphology of wild silk fiber was different with each other in spite of the similar degumming loss. Our result obtained after sodium carbonate degumming was similar to that of Kawahara and Nakajima (1992) after sodium hydrogen carbonate degumming. The reason why the surface morphology is different is not clear until now. Therefore, the systematic degumming research for A. pernyi cocoon filament will be needed to elucidate the relationship between degumming ratio and surface morphology of wild cocoon filament.

\section{Acknowledgement}

This study was supported by the Kyungpook National University Research Grant, 2010.

\section{References}

He J, Guo N, Cui S (2011) Structure and mechanical properties of electrospun tussah silk fibroin nanofibers: variations in processing parameters. Iranian Polymer Journal 20(9), 713724.

Kang P, Sung G, Kim K, Lee H, Kim M, Ji S, Hong I, Kweon H, Park K, Lee K, Sohn B (2011) Breeding of HanSaengJam a parental sex-limited larval marking strain suitable for spring rearing season. Int J Indust Entomol 23(1), 179-182.

Kang P, Kim K, Sung G, Kim M, Ji S, Kweon H, Park K, Shon B (2012b) Breeding of DaeBakJam a high silk yielding silkworm variety for spring rearing season. J Seric Entomol Sci $50(1), 1-4$.

Kang P, Kim K, Sung G, Kim M, Ji S, Kweon H, Park K, Shon B (2012b) Breeding of SooOkJam an artificial diet adaptable silkworm variety for spring rearing season. J Seric Entomol Sci 50(1), 5-9.

Kawahara Y, Nakajima S (1992) Surface and mechanical properties of wild silk fibers degummed with enzyme. J Seric Sci Jpn 61(5), 460-462.

Kweon HY, Lee KG, Lee YW, Yeo JH, Um IC (1998) Hydrolysis behaviour of Antheraea pernyi silk fiber treated with $\mathrm{HCl}$. Korean J Seric Sci 40(2), 163-168.

Kweon HY, Lee K, Woo S, Park YH (2002) Dissolution and 
characterization of Antheraea pernyi silk fibroin regenerated from zinc chloride solution. Korean J Seric Sci 44(2), 87-92.

Kweon HY, Lee K, Yeo J, Park YH (2003) Dissolution of Antheraea pernyi silk fiber and structure of regenerated fibroin from zinc nitrate solution. Korean J Seric Sci 45(2) ,121-125.

Kweon HY, Lee K, Park K, Kang S. Seok Y (2012) Degumming characteristics and color stability of GoldenSilk cocoon. Int J Indust Entomol 24(1), 1-5.

Lee K, Kweon HY, Yeo J, Woo S, Han S, Kim J (2011) Characterization of tyrosine-rich Antheraea pernyi silk fibroin hydrolysate. Int J Biol Macromol 48, 223-226.

Moon JY (2000) Wild silkworm; in Korean Sericultural Technology 100 Years History. Lee WC, Kim KY, Lee KG, Lee YW, Ryu KS, Lee SM, Jim BR (eds.) pp.284-286, Hanrimwon Co., Seoul.
Tao J, Jehan H, Jing W (1993) Studies on the chromogen of the tussah cocoon layer and its chromophoric mechanism. Science in China, series B 36(9), 1046-1051.

Um IC, Rico CW, Kweon HY, Kang MY (2011) Enhancement of lipid metabolism and antioxidant defense status in mice fed with high fat diet supplemented with Antheraea pernyi silk fibroin powder. Int J Indust Entomol 22(2), 95-100.

Woo S, Kweon HY, Um IC, Park YH (2000) Structural characteristics of regenerated Antheraea pernyi silk fibroin film treated with ethanol. Korean J Seric Sci 42(2), 114-119.

Yan S, Zhao C, Wu X, Zhang Q, Li M (2010) Gelation behavior of Antheraea pernyi silk fibroin. Science China Chemistry 53(3), 535-541.

Zhao C, Wu X, Zhang Q, Yan S, Li M (2011) Enzymatic degradation of Antheraea pernyi silk fibroin 3D scaffolds and fibers. Int J Biol Macromol 48(2), 249-255. 